\title{
Clinical Observations Sponsor Defined Identifier
}

National Cancer Institute

\section{Source}

National Cancer Institute. Clinical Observations Sponsor Defined Identifier. NCI

Thesaurus. Code C119811.

One or more sponsor defined characters used to identify, name, or characterize the clinical observation. 